Publ. Mat. 47 (2003), 415-440

\title{
MEAN DIRECTIONALLY CURVED LINES ON SURFACES IMMERSED IN $\mathbb{R}^{4}$
}

\author{
Luis FERnANDO MELLO
}

\begin{abstract}
The notion of principal configuration of immersions of surfaces into $\mathbb{R}^{3}$, due to Sotomayor and Gutierrez [16] for lines of curvature and umbilics, is extended to that of mean directional configuration for immersed surfaces in $\mathbb{R}^{4}$. This configuration consists on the families of mean directionally curved lines, along which the second fundamental form points in the direction of the mean curvature vector, and their singularities, called here $H$-singularities.

The concepts of $H$-singularities and periodic mean directionally curved lines are studied here in detail. Also the notion of principal structural stability of immersions of surfaces into $\mathbb{R}^{3}$ is extended to that of mean directional structural stability, for the case of surfaces in $\mathbb{R}^{4}$. Sufficient conditions for immersions to be mean directional structurally stable are provided in terms of $H$-singularities, periodic mean directionally curved lines and the asymptotic behavior of all the other mean directionally curved lines.
\end{abstract}

\section{Introduction}

Principal curvatures, principal direction fields, their integral foliations and umbilic singularities are classical topics of the theory of surfaces immersed in $\mathbb{R}^{3}$. Nevertheless the global behavior for a large class of these geometric objects has been understood only recently with the introduction, by Gutierrez and Sotomayor, of the notion of structural stability and genericity, originated from differential equations and dynamical systems. Their original works $[\mathbf{1 6}]$ and $[\mathbf{1 0}]$ are also presented in $[\mathbf{1 1}]$.

The global behavior of other geometric structures of the theory of surfaces immersed in $\mathbb{R}^{3}$, such as asymptotic lines [4] and lines of mean curvature [7], is still the subject of current works.

2000 Mathematics Subject Classification. 53C03, 58F14.

Key words. Ellipse of curvature, minimal points, inflection points, normal curvature, structural stability.

This work is supported in part by CNPq-Brazil, Grant 476886/2001-5. 
General aspects of the curvature theory for surfaces in $\mathbb{R}^{4}$ are presented in the works of Forsyth [3], Wong [18], Little [12] and Asperti [1].

The ideas of Gutierrez and Sotomayor have also been applied to some aspects of this theory. Such as the description of generic singularities of asymptotic lines [5], the study of principal lines on minimal surfaces [8], and the analysis of generic singularities of lines of curvature, [9] and [15]. Recently the global behavior of lines of axial curvature on surfaces immersed in $\mathbb{R}^{4}$ was studied by Garcia and Sotomayor [6].

The main feature of this paper is the study of the global generic structure of mean directionally curved lines on surfaces immersed in $\mathbb{R}^{4}$. Along these lines the second fundamental form points in the direction of the mean curvature vector. A review of properties of the first and second fundamental forms, the ellipse of curvature and related geometric objects is presented below. Afterwards, the main conclusions and the structure of this paper are formulated.

In this paper immersions are assumed to be $C^{\infty}$. Nevertheless the results can be adapted for $C^{r}$ immersions, $r \geq 4$. Let $\alpha: M \rightarrow \mathbb{R}^{4}$ be an immersion of a smooth and oriented surface into $\mathbb{R}^{4}$, which is endowed with the euclidean inner product $\langle\cdot, \cdot\rangle$ and oriented by a once for all fixed orientation. Denote respectively by $T M$ and $N M$ the tangent and the normal bundles of $\alpha$ and by $T_{p} M$ and $N_{p} M$ the respective fibers, i.e., the tangent and the normal planes at $p \in M$. Let $\left\{N_{1}, N_{2}\right\}$ be a frame of vector fields orthonormal to $\alpha$. Assume that $(u, v)$ is a positive chart of $M$ and that $\left\{\alpha_{u}, \alpha_{v}, N_{1}, N_{2}\right\}$ is a positive frame of $\mathbb{R}^{4}$. In such a chart $(u, v)$ the first fundamental form of $\alpha, I_{\alpha}$, is given by

$$
I=I_{\alpha}=\langle d \alpha, d \alpha\rangle=E d u^{2}+2 F d u d v+G d v^{2},
$$

where $E=\left\langle\alpha_{u}, \alpha_{u}\right\rangle, F=\left\langle\alpha_{u}, \alpha_{v}\right\rangle$ and $G=\left\langle\alpha_{v}, \alpha_{v}\right\rangle$. The second fundamental form of $\alpha, I I_{\alpha}$, is defined in terms of the $N M$-valued quadratic form

$$
I I=I I_{\alpha}=\left\langle d^{2} \alpha, N_{1}\right\rangle N_{1}+\left\langle d^{2} \alpha, N_{2}\right\rangle N_{2}=I I_{1, \alpha} N_{1}+I I_{2, \alpha} N_{2},
$$

where

$$
I I_{i}=I I_{i, \alpha}=e_{i} d u^{2}+2 f_{i} d u d v+g_{i} d v^{2},
$$

$e_{i}=\left\langle\alpha_{u u}, N_{i}\right\rangle, f_{i}=\left\langle\alpha_{u v}, N_{i}\right\rangle$, and $g_{i}=\left\langle\alpha_{v v}, N_{i}\right\rangle$, for $i=1,2$.

The following functions are associated to $\alpha$ (see $[\mathbf{1 2}])$ :

1. The mean curvature vector of $\alpha$

$$
H=H_{\alpha}=H_{1} N_{1}+H_{2} N_{2},
$$


where

$$
H_{i}=H_{i, \alpha}=\frac{E g_{i}-2 F f_{i}+G e_{i}}{2\left(E G-F^{2}\right)}, \quad \text { for } i=1,2 .
$$

2. The normal curvature of $\alpha$

$$
k_{N}=k_{N, \alpha}=\frac{E\left(f_{1} g_{2}-f_{2} g_{1}\right)-F\left(e_{1} g_{2}-e_{2} g_{1}\right)+G\left(e_{1} f_{2}-e_{2} f_{1}\right)}{2\left(E G-F^{2}\right)} .
$$

3. The resultant $\Delta$ of $I I_{1, \alpha}$ and $I I_{2, \alpha}$

$$
\Delta=\Delta_{\alpha}=\frac{1}{4\left(E G-F^{2}\right)}\left|\begin{array}{cccc}
e_{1} & 2 f_{1} & g_{1} & 0 \\
e_{2} & 2 f_{2} & g_{2} & 0 \\
0 & e_{1} & 2 f_{1} & g_{1} \\
0 & e_{2} & 2 f_{2} & g_{2}
\end{array}\right| .
$$

4. The normal curvature vector of $\alpha$ defined by $\eta: T M \rightarrow N M$, where $\eta(p, v)=\frac{I I(p, v)}{I(p, v)}$.

The image of the unitary circle $S^{1}$ of $T_{p} M$ by $\eta(p): T_{p} M \rightarrow N_{p} M$ describes an ellipse in $N_{p} M$ called ellipse of curvature of $\alpha$ at $p$ and denoted by $\varepsilon_{\alpha}(p)$. This ellipse may degenerate into a line segment, a circle or a point. The center of the ellipse of curvature is the mean curvature vector $H$ and their area is given by $\frac{\pi}{2}\left|k_{N}(p)\right|$. The map $\eta(p)$ restricted to $S^{1}$, being quadratic, is a double covering of the ellipse of curvature. Thus every point on the ellipse corresponds to two diametrically opposed points on the unitary tangent circle. The ellipse of curvature is invariant by rotations in the tangent and normal planes.

A point $p \in M$ is called a minimal point of $\alpha$ if $H(p)=0$ and it is called an inflection point of $\alpha$ if $\Delta(p)=k_{N}(p)=0$. It follows that $p \in M$ is an inflection point if and only if its ellipse of curvature is a radial line segment.

Based in the above results we have: from any well-defined continuous choice of points on the ellipse of curvature, continuous tangent direction fields may be constructed on $M$. If the construction fails for special points we say that they are singular points of the direction field. Consider the following construction.

The line through the mean curvature vector $H(p)$ meets the ellipse of curvature $\varepsilon_{\alpha}(p)$ at two points. This construction induces two orthogonal directions on $T_{p} M$. Making this construction for all $p \in M$ we define two direction fields on $M$, called $H$-direction fields. The singularities of these fields, called $H$-singularities, are the points where either $H=0$ (minimal 
points) or at which the ellipse of curvature becomes a radial line segment (inflection points). The set of $H$-singularities will be denoted by $S(\alpha)$.

A mean directionally curved line is a regular curve $\varphi:(a, b) \rightarrow M$ which at each of its points is tangent to an $H$-direction and it contains any regular curve with this property which intersects it. The mean directionally curved lines and their singularities are assembled into the mean directional configuration.

In this work, the notion of principal structural stability of immersions of surfaces into $\mathbb{R}^{3}$ is extended for the mean directional configurations of the case $\mathbb{R}^{4}$. Sufficient conditions are provided to extend to the present setting the Theorem on Structural Stability for Principal Configurations due to Gutierrez and Sotomayor [11]. Two local cases, treated in detail here, are essential for this extension: $H$-singularities with their separatrix structure and closed (i.e., the cycles or periodic) mean directionally curved lines.

This paper is organized as follows:

In Section 2 we analyse the differential equation of mean directionally curved lines in an arbitrary chart. It is shown that this differential equation fits into the class of quadratic or binary differential equations.

Section 3 is devoted to the analysis of $H$-singularities. For this purpose the differential equation of mean directionally curved lines is written in a Monge chart. The $H$-singularity condition is explicitly stated in terms of the coefficients of second order jet of the two functions which represent the immersion in a Monge chart. The condition of stability at $H$-singularity is expressed in an invariant form involving the third order jets.

In Section 4 the derivative of first return map along a mean directionally curved cycle is established. It consists of an integral involving geometric functions along the cycle.

In Section 5 the results presented in Sections 3 and 4 are put together to provide sufficient conditions for mean directional structural stability.

In Section 6 we analyse the case where the surface $M$ is immersed in $S^{3}$. In this case $H$-singularities only appear at inflection points. A correspondence between mean directionally curved lines on $M$ and lines of mean curvature on $\phi^{-1}(M)$ is established, where $\phi: \mathbb{R}^{3} \rightarrow S^{3}$ is the stereographic projection. Furthermore it is shown that, for surfaces in $S^{3}$, the quartic differential equation of lines of axial curvature factors into the quadratic differential equation of mean directionally curved lines and the quadratic differential equation of asymptotic lines. 


\section{Differential equation for mean directionally curved lines} by

$$
\eta=\mu H,
$$

where $\mu \in \mathbb{R}$. Eliminating $\mu$ in (2.1) we have a quadratic differential equation of the form

$$
A(u, v) d u^{2}+2 B(u, v) d u d v+C(u, v) d v^{2}=0,
$$

where

$$
\begin{aligned}
& A=A(u, v)=\left(e_{1} g_{2}-e_{2} g_{1}\right) E+2\left(e_{2} f_{1}-e_{1} f_{2}\right) F, \\
& B=B(u, v)=\left(f_{1} g_{2}-f_{2} g_{1}\right) E+\left(e_{2} f_{1}-e_{1} f_{2}\right) G, \\
& C=C(u, v)=2\left(f_{1} g_{2}-f_{2} g_{1}\right) F+\left(e_{2} g_{1}-e_{1} g_{2}\right) G .
\end{aligned}
$$

The $H$-singularities are determined by $A=B=C=0$ in (2.2). But it is immediate that the equation $E C=2 F B-G A$ holds.

We have established the following proposition.

Proposition 2.1. Let $\alpha: M \rightarrow \mathbb{R}^{4}$ be an immersion of a smooth and oriented surface into $\mathbb{R}^{4}$. With the above notations we have:

1. The differential equation of mean directionally curved lines is given by (2.2).

2. The $H$-singularities of $\alpha$ are given by $A=B=0$, where $A$ and $B$ are defined in (2.3) and (2.4) respectively.

Proposition 2.2. $A(p)=B(p)=0$ if and only if $p$ is either a minimal point or an inflection point of $M$.

Proof: It is enough to prove the proposition for the isothermic coordinates where $E=G=\lambda^{2}$ and $F=0$. In this case equation (2.2) has the form

$$
A_{1}(u, v) d u^{2}+2 B_{1}(u, v) d u d v-A_{1}(u, v) d v^{2}=0,
$$

where

$$
A_{1}=e_{1} g_{2}-e_{2} g_{1},
$$

and

$$
B_{1}=f_{1}\left(e_{2}+g_{2}\right)-f_{2}\left(e_{1}+g_{1}\right) .
$$


Thus

$$
0=A_{1} f_{1}+B_{1} g_{1}=\left(e_{1}+g_{1}\right)\left(f_{1} g_{2}-f_{2} g_{1}\right)
$$

and

$$
0=A_{1} f_{2}+B_{1} g_{2}=\left(e_{2}+g_{2}\right)\left(f_{1} g_{2}-f_{2} g_{1}\right) .
$$

If $f_{1} g_{2}-f_{2} g_{1} \neq 0$ then $e_{1}+g_{1}=e_{2}+g_{2}=0$ and this implies that $H(p)=0$, i.e., $p$ is a minimal point. If $f_{1} g_{2}-f_{2} g_{1}=0$ then $e_{1} f_{2}-e_{2} f_{1}=$ $e_{1} g_{2}-e_{2} g_{1}=f_{1} g_{2}-f_{2} g_{1}=0$ and this implies that $p$ is an inflection point. The reciprocal is immediate.

From the Proposition 2.2, $S(\alpha)=S_{1}(\alpha) \cup S_{2}(\alpha)$, where $S_{1}(\alpha)$ is the set of minimal points of $M$ and $S_{2}(\alpha)$ is the set of inflection points of $M$.

The differential equation (2.2) is also obtained equivalently by

$$
\operatorname{Jac}\left\{\operatorname{Jac}\left(I I_{1}, I I_{2}\right), I\right\}=0,
$$

where

$$
\operatorname{Jac}(\cdot, \cdot)=\frac{\partial(\cdot, \cdot)}{\partial(d u, d v)} .
$$

Equation (2.6) suggests the definition of the quadratic form

$$
\begin{aligned}
J=J_{\alpha} & =\operatorname{Jac}\left(I I_{1}, I I_{2}\right) \\
& =\left(e_{1} f_{2}-e_{2} f_{1}\right) d u^{2}+\left(e_{1} g_{2}-e_{2} g_{1}\right) d u d v+\left(f_{1} g_{2}-f_{2} g_{1}\right) d v^{2} .
\end{aligned}
$$

Denote by $\lambda_{1}(p)$ and $\lambda_{2}(p)$, with $\lambda_{1}(p) \leq \lambda_{2}(p)$, the extreme values of $J$ as $w$ ranges on the unitary circle of $T_{p} M$. These extreme values are the roots of

$$
\lambda^{2}-2 k_{N} \lambda+\Delta=0 .
$$

In fact, for $w=a \frac{\partial}{\partial u}+b \frac{\partial}{\partial v}, J(w, w)$ restricted to $I(w, w)=1$ is stationary if and only if, for some (Lagrange multiplier) $\lambda$,

$$
\frac{\partial}{\partial a}(J(w, w))=\lambda \frac{\partial}{\partial a}(I(w, w)) \quad \text { and } \quad \frac{\partial}{\partial b}(J(w, w))=\lambda \frac{\partial}{\partial b}(I(w, w)) \text {. }
$$

Performing the differentiation and eliminating $a$ and $b, \lambda$ will satisfy the above equation. Thus the unitary vectors $\pm e_{1}(p)$ and $\pm e_{2}(p)$, at which the extreme values $\lambda_{1}(p)$ and $\lambda_{2}(p)$ are attained, are well defined. They are mutually orthogonal for $p$ outside the set $S(\alpha)$, at which $\lambda_{1}(p)=$ $\lambda_{2}(p)$. Now, performing the differentiation of (2.7) and eliminating $\lambda$ gives that, in the chart $(u, v)$, the components $a$ and $b$ of $\pm e_{1}(p)$ and $\pm e_{2}(p)$ satisfies

$$
A(u, v) a^{2}+2 B(u, v) a b+C(u, v) b^{2}=0,
$$


i.e., the differential equation of mean directionally curved lines (2.2). We define $l_{H}(\alpha)=\mathbb{R}\left( \pm e_{1}\right)$ and $L_{H}(\alpha)=\mathbb{R}\left( \pm e_{2}\right)$.

If $p \notin S(\alpha)$ then $\left(B^{2}-A C\right)(p)>0$. This implies the existence of two orthogonal solutions of the differential equation of mean directionally curved lines (2.2). Thus in a neighborhood of this point there exist two families of orthogonal curves. Under the orientability hypothesis it is possible to extend these lines to the whole $M$. Each family defines a foliation, denoted by $F_{H}(\alpha)$ and $f_{H}(\alpha)$ respectively, on the surface without the $H$-singularities. The foliation $F_{H}(\alpha)\left(f_{H}(\alpha)\right)$ is associated to $L_{H}(\alpha)$ $\left(l_{H}(\alpha)\right)$. Each isolated $H$-singularity defines an isolated singularity of both foliations.

The mean directional configuration of an immersion $\alpha: M \rightarrow \mathbb{R}^{4}$ is the triple

$$
\mathcal{H}(\alpha)=\left\{S(\alpha), F_{H}(\alpha), f_{H}(\alpha)\right\} .
$$

It synthesizes the qualitative properties of the foliations $F_{H}(\alpha)$ and $f_{H}(\alpha)$ and represents the way their lines approach the $H$-singularity set. It is a natural analog of the principal configuration of an immersion $\alpha: M \rightarrow \mathbb{R}^{3}$.

\section{Mean directional configurations near $\boldsymbol{H}$-singularities}

Let $p \in M$ be an $H$-singularity. We say that $p$ is a transversal $H$-singularity if

$$
J(A, B)(p)=\frac{\partial(A, B)}{\partial(u, v)}(p) \neq 0,
$$

where $A$ and $B$ are defined in (2.3) and (2.4) respectively. This condition means that the curves $A=0$ and $B=0$, whose intersection defines the $H$-singularities, are regular and meet transversally at $p$. It follows that transversal $H$-singularities are isolated.

Consider the surface $M$ in a Monge chart, i.e., the surface $M$ is the graph of the map $\alpha(u, v)=(u, v, S(u, v), R(u, v))$, where $S$ and $R$ are $C^{\infty}$ functions defined on a neighborhood $U \subset \mathbb{R}^{2}$ of $(0,0)$ with the conditions

$$
S(0,0)=R(0,0)=S_{u}(0,0)=R_{u}(0,0)=S_{v}(0,0)=R_{v}(0,0)=0 .
$$

For each point $\alpha(u, v) \in M$ the tangent plane $T_{\alpha(u, v)} M$ is generated by $\left\{\alpha_{u}(u, v)=\left(1,0, S_{u}(u, v), R_{u}(u, v)\right), \alpha_{v}(u, v)=\left(0,1, S_{v}(u, v), R_{v}(u, v)\right)\right\}$ and the normal plane $N_{\alpha(u, v)} M$ is generated by $\left\{\bar{N}_{1}, \bar{N}_{2}\right\}$, where 


$$
\bar{N}_{1}=\left(-S_{u},-S_{v}, 1,0\right)
$$

and

$$
\begin{aligned}
\bar{N}_{2}= & -R_{u}\left(1+S_{v}{ }^{2}\right)+S_{u} S_{v} R_{v} \\
& \left.-R_{v}\left(1+S_{u}{ }^{2}\right)+S_{u} S_{v} R_{v},-S_{u} R_{u}-S_{v} R_{v}, 1+S_{u}{ }^{2}+S_{v}{ }^{2}\right) .
\end{aligned}
$$

Therefore

$$
\begin{aligned}
& E=\left\langle\alpha_{u}, \alpha_{u}\right\rangle, \quad F=\left\langle\alpha_{u}, \alpha_{v}\right\rangle, \quad G=\left\langle\alpha_{v}, \alpha_{v}\right\rangle, \\
& e_{i}=\left\langle\alpha_{u u}, N_{i}\right\rangle, \quad f_{i}=\left\langle\alpha_{u v}, N_{i}\right\rangle, \quad g_{i}=\left\langle\alpha_{v v}, N_{i}\right\rangle,
\end{aligned}
$$

where

$$
N_{i}=\frac{\bar{N}_{i}}{\left\|\bar{N}_{i}\right\|},
$$

for $i=1,2$.

Write the Taylor's expansion of the functions $S$ and $R$ near $(0,0)$

(3.1) $S(u, v)=\frac{s_{20}}{2} u^{2}+s_{11} u v+\frac{s_{02}}{2} v^{2}+\frac{a}{6} u^{3}+\frac{d}{2} u^{2} v+\frac{b}{2} u v^{2}+\frac{c}{6} v^{3}+O(4)$,

(3.2) $R(u, v)=\frac{r_{20}}{2} u^{2}+r_{11} u v+\frac{r_{02}}{2} v^{2}+\frac{\bar{a}}{6} u^{3}+\frac{\bar{d}}{2} u^{2} v+\frac{\bar{b}}{2} u v^{2}+\frac{\bar{c}}{6} v^{3}+O(4)$.

Thus the coefficients of the first and the second fundamental forms in a Monge chart are given by

$$
E=1+O(2), \quad F=O(2), \quad G=1+O(2),
$$

$e_{1}=s_{20}+a u+d v+O(2), f_{1}=s_{11}+d u+b v+O(2), g_{1}=s_{02}+b u+c v+O(2)$,

$e_{2}=r_{20}+\bar{a} u+\bar{d} v+O(2), f_{2}=r_{11}+\bar{d} u+\bar{b} v+O(2), g_{2}=r_{02}+\bar{b} u+\bar{c} v+O(2)$. 
Define

$$
\begin{aligned}
J & =s_{02} r_{20}-s_{20} r_{02}, \\
K & =s_{02} \bar{a}+r_{20} b-s_{20} \bar{b}-r_{02} a, \\
L & =s_{02} \bar{d}+r_{20} c-s_{20} \bar{c}-r_{02} d, \\
M & =s_{11}\left(r_{20}+r_{02}\right)-r_{11}\left(s_{20}+s_{02}\right), \\
N & =s_{11}(\bar{a}+\bar{b})-r_{11}(a+b)+\left(r_{20}+r_{02}\right) d-\left(s_{20}+s_{02}\right) \bar{d}, \\
P & =s_{11}(\bar{c}+\bar{d})-r_{11}(c+d)+\left(r_{20}+r_{02}\right) b-\left(s_{20}+s_{02}\right) \bar{b} .
\end{aligned}
$$

The differential equation of mean directionally curved lines (2.2) in a Monge chart has the form

$$
C(u, v) d v^{2}+2 B(u, v) d u d v+A(u, v) d u^{2}=0,
$$

where

$$
\begin{aligned}
& C(u, v)=J+K u+L v+Q_{1}(u, v), \\
& B(u, v)=M+N u+P v+Q_{2}(u, v), \\
& A(u, v)=-J-K u-L v+Q_{3}(u, v),
\end{aligned}
$$

and $Q_{1}, Q_{2}$ and $Q_{3}$ are of order $O(2)$.

From (3.3) the condition for $(0,0)$ to be an $H$-singularity is that

$$
J=s_{02} r_{20}-s_{20} r_{02}=0
$$

and

$$
M=s_{11}\left(r_{20}+r_{02}\right)-r_{11}\left(s_{20}+s_{02}\right)=0 .
$$

Consider the tangent projective bundle $P M=\{T M-\{0\} /\{v=r w$, $r \neq 0\}\}$ of $M$. The natural projection is given by $\pi: P M \rightarrow M$. We put $p=d v / d u$ and $q=d u / d v$. Thus $P M$ can be parametrized by charts $(u, v ; p)$ and $(u, v ; q)$. On $P M$ consider the surface $W$ defined by the differential equation of mean directionally curved lines. In coordinates $(u, v ; p)$ this surface is defined by $T^{-1}(0)$ where, from (3.3),

$$
\begin{aligned}
T(u, v ; p)= & \left(J+K u+L v+Q_{1}\right) p^{2} \\
& +2\left(M+N u+P v+Q_{2}\right) p-\left(J+K u+L v+Q_{3}\right) .
\end{aligned}
$$

If $(0,0)$ is an $H$-singularity then $(0,0 ; p) \in W$, for all $p$. Furthermore the surface $W$ is smooth in a neighborhood of the $p$-axis if and only if $(0,0)$ is a transversal $H$-singularity, according to [11] and [2]. 
Suppose that $(0,0)$ is a transversal $H$-singularity. In this case consider the following vector field on $W$

(3.5) $X(u, v ; p)=\left(T_{p}(u, v ; p), p T_{p}(u, v ; p),-\left(T_{u}(u, v ; p)+p T_{v}(u, v ; p)\right)\right)$.

The vector field $X$ has generically either one or three singularities on $p$-axis, which are of type saddle or node, according to [11].

Proposition 3.1. Suppose the case where $K=0$. With the above construction $p \in M$ is a transversal $H$-singularity if and only if

$$
N L \neq 0 \text {. }
$$

Proof: The condition of transversality is given by $2(K P-N L) \neq 0$, which is equivalent to (3.6).

Proposition 3.2. Suppose the case where $K=0$. Consider a transversal $H$-singularity as above. Then we have:

1. If

$$
\frac{N}{L}>\frac{1}{2}\left[\left(\frac{P}{L}\right)^{2}+1\right],
$$

then the $H$-singularity is of type $S_{1}$ (see Figure 1).

2. If

$$
\frac{1}{2}\left[\left(\frac{P}{L}\right)^{2}+1\right]>\frac{N}{L}>0, \quad 2 N \neq L,
$$

then the $H$-singularity is of type $S_{2}$ (see Figure 1).

3. If

$$
\frac{N}{L}<0
$$

then the $H$-singularity is of type $S_{3}$ (see Figure 1).

Proof: The singularities of the vector field $X(3.5)$ on the $p$-axis are given by

But

$$
\varphi(p)=T_{u}(0,0 ; p)+p T_{v}(0,0 ; p)=0 \text {. }
$$

whose roots are

$$
0=\varphi(p)=p\left[L p^{2}+2 P p+2 N-L\right]
$$

$$
\begin{aligned}
& p_{0}=0 \\
& p_{1}=\frac{-P}{L}+\sqrt{\left(\frac{P}{L}\right)^{2}-\frac{2 N}{L}+1}
\end{aligned}
$$


and

$$
p_{2}=\frac{-P}{L}-\sqrt{\left(\frac{P}{L}\right)^{2}-\frac{2 N}{L}+1} .
$$

The Jacobian matrix of the vector field $X$ at $(0,0 ; p)$ is given by

$$
\left(\begin{array}{ccc}
2 N & 2(L p+P) & 0 \\
2 N p & 2 p(L p+P) & 0 \\
* & * & -\left(3 L p^{2}+4 P p+2 N-L\right)
\end{array}\right) .
$$

Therefore the Jacobian determinant of $X$ at $p_{0}=0$ is $J X(0)=2 N(L-$ $2 N)$. Thus, we have

$$
\frac{J X(0)}{L^{2}}=\frac{2 N}{L}\left(1-\frac{2 N}{L}\right) .
$$

The vector field $X$ has:

1. One singularity which is a saddle if (3.7) holds. In fact, in this case we have

$$
\frac{2 N}{L}>\left(\frac{P}{L}\right)^{2}+1
$$

and this implies that $J X(0)$ is negative and the only singular point of $X$ is a saddle.

2. Three singularities which are one node and two saddles if (3.8) holds. In fact, in this case there are two possibilities at $p_{0}=0$ :

(a) If

$$
1>\frac{2 N}{L}>0
$$

then $J X(0)$ is positive and the origin is a hyperbolic node.

(b) If

$$
\frac{2 N}{L}>1
$$

then $J X(0)$ is negative and the origin is a saddle.

As any singular point of $X$ can be taken to the origin by appropriate change of coordinates, the analysis above imply that one of the singularities of $X$, that in the middle, is a node and the other two are saddles.

3. Three singularities which are three saddles if (3.9) holds. In fact, in this case we have

$$
\frac{2 N}{L}<0,
$$

and this implies that $J X(0)$ is negative and the origin is a saddle. The singularities $p_{1}$ and $p_{2}$ are also saddle points. 
The local configurations of the $H$-singularities $S_{1}, S_{2}$ and $S_{3}$ illustrated in the Figure 1 are obtained by projection onto the $u v$-plane of the local configurations of the above singularities, according to $[\mathbf{1 1}]$.

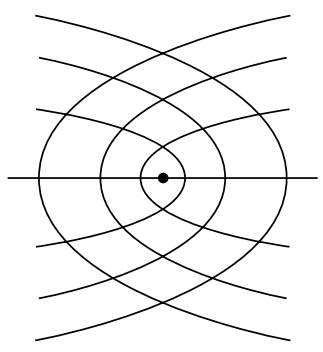

Type $S_{1}$

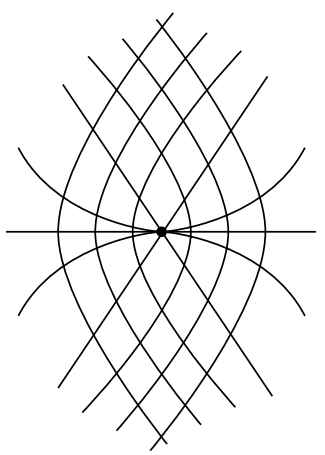

Type $S_{2}$

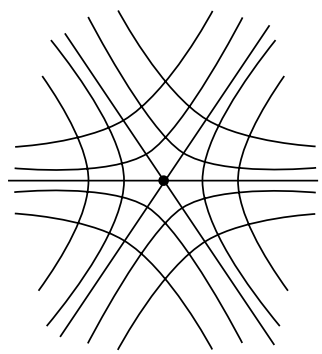

Type $S_{3}$

FIGURE 1. $H$-singularities of $S$-type.

Remark 3.3. The index $i=1,2,3$ of $S_{i}$ denotes the number of separatrices of the $H$-singularity. These are mean directionally curved lines which approach of the $H$-singularity and which separate regions of different patterns of approach to it. Figure 1 shows the local mean directional configuration of three different types of $H$-singularity denoted by $S_{1}, S_{2}$ and $S_{3}$ and called $H$-singularity of $S$-type.

Proposition 3.4. Suppose that $p \in M$ is an $H$-singularity with $H(p)=$ 0 and $k_{N}(p) \neq 0$. Then (3.1) and (3.2) can be written as

$$
\begin{aligned}
& S(u, v)=\frac{A}{2}\left(u^{2}-v^{2}\right)+\frac{a}{6} u^{3}+\frac{d}{2} u^{2} v+\frac{b}{2} u v^{2}+\frac{c}{6} v^{3}+O(4), \\
& R(u, v)=C u v+\frac{\bar{a}}{6} u^{3}+\frac{\bar{d}}{2} u^{2} v-\frac{\bar{a}}{2} u v^{2}+\frac{\bar{c}}{6} v^{3}+O(4) .
\end{aligned}
$$

Proof: Through an appropriate rotation in the normal plane it is possible to write $r_{20}=r_{02}=0$. This implies that $s_{11}=0$. Through an appropriate rotation in the $u v$-plane it is possible to write $\bar{b}=-\bar{a}$. Substituting these expressions in (3.1) and (3.2) the proposition is proved. 
From (3.10) and (3.11) we have

$$
\begin{aligned}
& J=0, \\
& K=0, \\
& L=-A(\bar{c}+\bar{d}), \\
& M=0, \\
& N=-C(a+b), \\
& P=-C(c+d) .
\end{aligned}
$$

Proposition 3.5. Suppose that $p \in M$ is an $H$-singularity which is an inflection point and that $H(p) \neq 0$. Then (3.1) and (3.2) can be written as

$$
\begin{aligned}
& S(u, v)=\frac{A}{2}\left(u^{2}+v^{2}\right)+B u v+O(3), \\
& R(u, v)=\frac{\bar{a}}{6} u^{3}+\frac{\bar{d}}{2} u^{2} v+\frac{\bar{a}}{2} u v^{2}+\frac{\bar{c}}{6} v^{3}+O(4) .
\end{aligned}
$$

Proof: Through an appropriate rotation in the normal plane it is possible to write $r_{20}=r_{02}=r_{11}=0$. Through an appropriate rotation in the $u v$-plane it is possible to write $\bar{b}=\bar{a}$. Substituting these expressions in (3.1) and (3.2) the proposition is proved.

From (3.12) and (3.13) we have

$$
\begin{aligned}
J & =0, \\
K & =0, \\
L & =A(\bar{d}-\bar{c}), \\
M & =0, \\
N & =2(\bar{a} B-A \bar{d}), \\
P & =B(\bar{c}+\bar{d})-2 A \bar{a} .
\end{aligned}
$$

Remark 3.6. As a consequence of Propositions 3.1, 3.2, 3.4 and 3.5 we have the description of $H$-singularities of $S$-type in terms of the coefficients of third order jets. 


\section{Mean directionally curved cycles}

In terms of geometric invariants, here is established the formula of the first derivative of the first return map of a periodic mean directionally curved line, called here mean directionally curved cycle. This first return map, denoted by $\pi$, is called holonomy in foliation theory and Poincaré map in dynamical systems.

A mean directionally curved cycle is called hyperbolic if the first derivative of the first return map at the fixed point is different from one.

Let $\gamma: I \rightarrow M$ be a mean directionally curved cycle of the foliation $f_{H}(\alpha)$, parametrized by arc length $s$ and of length $L$. We take $\left\{T_{1}(s)=\gamma^{\prime}(s), T_{2}(s)\right\}$ an orthonormal frame of $T_{\gamma(s)} M,\left\{N_{1}(s), N_{2}(s)\right\}$ an orthonormal frame of $N_{\gamma(s)} M$ such that $\left\{T_{1}, T_{2}, N_{1}, N_{2}\right\}$ be a positive frame of $\mathbb{R}^{4}$. We choose the frame $\left\{N_{1}(s), N_{2}(s)\right\}$ with the following properties

$$
\eta\left(\gamma(s), T_{1}(s)\right)=a(s) H_{1}(s) N_{1}(s)
$$

and

$$
\eta\left(\gamma(s), T_{2}(s)\right)=b(s) H_{1}(s) N_{1}(s) .
$$

This means that the functions $a$ and $b$ are linked by a relation of the form $a+b \equiv 2$.

The tangent projection of the vector field $\gamma^{\prime \prime}(s)$ along $\gamma$ is given by $k_{g}(s) T_{2}(s)$. Define the geodesic curvature of $\gamma$ by $k_{g}(s)=\left\langle\gamma^{\prime \prime}(s), T_{2}(s)\right\rangle$. Now the normal component of $\gamma^{\prime \prime}(s)$ is given by $\eta\left(\gamma(s), T_{1}(s)\right)$. Define the geodesic torsion vector by $\tau_{g}=\tau_{g, 1} N_{1}+\tau_{g, 2} N_{2}$ and the normal torsion of the frame $\left\{N_{1}, N_{2}\right\}$ by $\tau_{n}=\left\langle N_{1}^{\prime}, N_{2}\right\rangle$.

In a similar way as in the case of surfaces in $\mathbb{R}^{3}$ (see $[\mathbf{1 7}$, p. 131]), we will obtain a system of equations of the Darboux frame $\left\{T_{1}(s), T_{2}(s), N_{1}(s), N_{2}(s)\right\}$ associated to $\gamma$ :

$$
\begin{aligned}
& T_{1}^{\prime}(s)=k_{g}(s) T_{2}(s)+\eta\left(\gamma(s), T_{1}(s)\right)=k_{g}(s) T_{2}(s)+a(s) H_{1}(s) N_{1}(s) ; \\
& T_{2}^{\prime}(s)=-k_{g}(s) T_{1}(s)+\tau_{g}(s)=-k_{g}(s) T_{1}(s)+\tau_{g, 1}(s) N_{1}(s)+\tau_{g, 2}(s) N_{2}(s) ; \\
& N_{1}^{\prime}(s)=-a(s) H_{1}(s) T_{1}(s)-\tau_{g, 1}(s) T_{2}(s)+\tau_{n}(s) N_{2}(s) ; \\
& N_{2}^{\prime}(s)=-\tau_{g, 2}(s) T_{2}(s)-\tau_{n}(s) N_{1}(s) .
\end{aligned}
$$

Here $H=H_{1} N_{1}$ is the mean curvature vector.

At the point $\gamma(s)$, the intersection of the surface $M$ with the hyperplane generated by $\left\{T_{2}(s), N_{1}(s), N_{2}(s)\right\}$ is a curve $\Gamma_{s}$, tangent to $T_{2}(s)$ 
at $\gamma(s)$. This curve can be parametrized by

$$
\Gamma_{s}(t)=\gamma(s)+t T_{2}(s)+V_{1}(s, t) N_{1}(s)+V_{2}(s, t) N_{2}(s)
$$

and its curvature is given by $\eta\left(\gamma(s), T_{2}(s)\right)$. Thus we have the following expressions

$$
V_{1}(s, t)=\frac{b(s) H_{1}(s)}{2} t^{2}+\bar{A}(s, t) \frac{t^{3}}{6}+O(4)
$$

and

$$
V_{2}(s, t)=\bar{B}(s, t) \frac{t^{3}}{6}+O(4),
$$

with $\bar{A}(s)=\bar{A}(s, 0)$ and $\bar{B}(s)=\bar{B}(s, 0)$.

Lemma 4.1. Let $\gamma$ be a mean directionally curved cycle of the foliation $f_{H}(\alpha)$, parametrized by arc length $s$ and of length $L$. We take the orthonormal positive Darboux frame along $\gamma$. Then the expression

$$
\begin{array}{r}
\alpha(s, t)=\gamma(s)+t T_{2}(s)+\left[\frac{b(s) H_{1}(s)}{2} t^{2}+\bar{A}(s, t) \frac{t^{3}}{6}+O(4)\right] N_{1}(s) \\
+\left[\bar{B}(s, t) \frac{t^{3}}{6}+O(4)\right] N_{2}(s)
\end{array}
$$

defines a local chart of $M, L$ periodic in $s$, in a neighborhood of $\gamma$.

Proof: The lemma follows from the Inverse Function Theorem applied to the map

$$
\alpha\left(s, t, v_{1}, v_{2}\right)=\gamma(s)+t T_{2}(s)+v_{1} N_{1}(s)+v_{2} N_{2}(s),
$$

which defines a tubular neighborhood of $\gamma$.

Differentiation of the equation (4.1) gives that

$$
E(s, 0)=G(s, 0)=1, \quad F(s, 0)=0,
$$

$$
\begin{array}{ll}
e_{1}(s, 0)=a(s) H_{1}(s), & f_{1}(s, 0)=\tau_{g, 1}(s), \quad g_{1}(s, 0)=b(s) H_{1}(s), \\
e_{2}(s, 0)=g_{2}(s, 0)=0, & f_{2}(s, 0)=\tau_{g, 2}(s) .
\end{array}
$$

We note that

$$
k_{N}(s)=k_{N}(s, 0)=\tau_{g, 2}(s)(a(s)-b(s)) H_{1}(s)
$$

and

$$
\Delta(s)=\Delta(s, 0)=-\left(\tau_{g, 2}(s)\right)^{2} a(s) b(s)\left(H_{1}(s)\right)^{2} .
$$


Since $H_{1}(s) \neq 0$ and $(a(s))^{2}+(b(s))^{2} \neq 0$, for all $s \in[0, L]$, we have that $\tau_{g, 2}(s) \neq 0$, for all $s \in[0, L]$.

Lemma 4.2. Let $\gamma$ be a mean directionally curved line and consider the chart $(s, t)$ given in (4.1). Then the orthonormal frame $\left\{N_{1}, N_{2}\right\}$ of the normal bundle satisfies the following equations

$$
\left(N_{1}\right)_{t}(s, 0)=-\tau_{g, 1}(s) T_{1}(s)-b(s) H_{1}(s) T_{2}(s)+a_{12}^{3}(s) N_{2}(s)
$$

and

$$
\left(N_{2}\right)_{t}(s, 0)=-\tau_{g, 2}(s) T_{1}(s)-a_{12}^{3}(s) N_{1}(s),
$$

where $a_{12}^{3}(s)=a_{12}^{3}(s, 0)=\left\langle\left(N_{1}\right)_{t}(s, 0), N_{2}(s, 0)\right\rangle$ is the normal torsion of the frame $\left\{N_{1}, N_{2}\right\}$ associated to the mean directionally curved line orthogonal to $\gamma$ at the point $\gamma(s)$.

Proof: In a chart $(s, t)$ the following equations hold

$$
\left(N_{1}\right)_{t}=\frac{g_{1} F-f_{1} G}{E G-F^{2}} \alpha_{s}+\frac{f_{1} F-g_{1} E}{E G-F^{2}} \alpha_{t}+a_{12}^{3} N_{2}
$$

and

$$
\left(N_{2}\right)_{t}=\frac{g_{2} F-f_{2} G}{E G-F^{2}} \alpha_{s}+\frac{f_{2} F-g_{2} E}{E G-F^{2}} \alpha_{t}-a_{12}^{3} N_{1} .
$$

Using (4.2) the lemma is proved.

Direct calculation shows that the following equations hold

$$
\begin{aligned}
& E_{t}(s, 0)=-2 k_{g}, \quad F_{t}(s, 0)=G_{t}(s, 0)=0, \\
& \left(e_{1}\right)_{t}(s, 0)=\tau_{g, 1}^{\prime}-\tau_{g, 2} \tau_{n}-k_{g} H_{1}(a+b), \\
& \left(f_{1}\right)_{t}(s, 0)=\left(b H_{1}\right)^{\prime}+k_{g} \tau_{g, 1}+\tau_{g, 2} a_{12}^{3}, \\
& \left(g_{1}\right)_{t}(s, 0)=\bar{A} \\
& \left(e_{2}\right)_{t}(s, 0)=\tau_{g, 1} \tau_{n}+\tau_{g, 2}^{\prime}-a H_{1} a_{12}^{3}, \\
& \left(f_{2}\right)_{t}(s, 0)=k_{g} \tau_{g, 2}-\tau_{g, 1} a_{12}^{3}, \\
& \left(g_{2}\right)_{t}(s, 0)=\bar{B}-b H_{1} a_{12}^{3} .
\end{aligned}
$$

Lemma 4.3. The function $\bar{B}$ introduced in (4.1) is given by

$$
\bar{B}=2\left(H_{2}\right)_{t}+H_{1} a_{12}^{3}(a+b)-\tau_{g, 1} \tau_{n}-\tau_{g, 2}^{\prime} .
$$


Proof: In the coordinates $(s, t)$ we have

$$
H_{2}=\frac{E g_{2}-2 F f_{2}+G e_{2}}{2\left(E G-F^{2}\right)} .
$$

Differentiating $\mathrm{H}_{2}$ and using the above equations, the lemma is proved.

Theorem 4.4. Let $\gamma$ be a mean directionally curved cycle of the foliation $f_{H}(\alpha)$, parametrized by arc length $s$ and of length $L$. Then the derivative of the first return map is given by

$\pi^{\prime}(0)=\exp \left[\frac{1}{2} \int_{0}^{L}\left[\frac{a(s)\left(\left(H_{2}\right)_{t}(s)+H_{1}(s) a_{12}^{3}(s)\right)-\tau_{g, 1}(s) \tau_{n}(s)}{\tau_{g, 2}(s)}\right] d s\right]$.

Proof: The derivative of the first return map satisfies the following linear differential equation

$$
\frac{d}{d s}\left(\frac{d t}{d t_{0}}\right)=-\frac{1}{2 B} \frac{\partial A}{\partial t} \frac{d t}{d t_{0}} .
$$

Therefore

$$
\pi^{\prime}(0)=\exp \left[\int_{0}^{L} \frac{-A_{t}(s, 0)}{2 B(s, 0)} d s\right]
$$

where the functions $A$ and $B$ are given in (2.3) and (2.4). Using (4.2), (4.3) and (4.4) we have

$$
2 B(s, 0)=-2 H_{1}(s) \tau_{g, 2}(s)(a(s)+b(s)),
$$

and

$$
A_{t}(s, 0)=H_{1}(s)\left[a(s) \bar{B}(s)-b(s)\left(\tau_{g, 1}(s) \tau_{n}(s)+\tau_{g, 2}^{\prime}(s)\right)\right] .
$$

Substituting (4.5), (4.8) and (4.9) in (4.7) the theorem follows.

Remark 4.5. The corresponding formula for the first derivative of the first return map when $\gamma$ is a mean directionally curved cycle of the foliation $F_{H}(\alpha)$ is given by

$$
\pi^{\prime}(0)=\exp \left[\frac{1}{2} \int_{0}^{L}\left[\frac{b(s)\left(\left(H_{2}\right)_{t}(s)+H_{1}(s) a_{12}^{3}(s)\right)-\tau_{g, 1}(s) \tau_{n}(s)}{\tau_{g, 2}(s)}\right] d s\right] .
$$


Proposition 4.6. Let $\alpha: M \rightarrow \mathbb{R}^{4}$ be an immersion of a smooth and oriented surface into $\mathbb{R}^{4}$, and let $\gamma$ be a mean directionally curved cycle of the foliation $f_{H}(\alpha)$, parametrized by arc length $s$ and of length $L$. Consider a chart $(s, t)$ as in the Lemma 4.1 and consider the deformation

$$
\beta_{\epsilon}(s, t)=\beta(\epsilon, s, t)=\alpha(s, t)+\epsilon\left[\frac{a(s) \tau_{g, 2}(s)}{6} t^{3}\right] \delta(t) N_{2}(s),
$$

where $\delta=1$ in neighborhood of $t=0$, with small support and $a \not \equiv 0$. Then $\gamma$ is a mean directionally curved cycle of $\beta_{\epsilon}$, for all $\epsilon \geq 0$ small, and $\gamma$ is a hyperbolic mean directionally curved cycle for $\beta_{\epsilon}, \epsilon \neq 0$.

Proof: From direct calculation with the deformation $\beta_{\epsilon}$ it follows that $\gamma$ is a mean directionally curved cycle for all $\beta_{\epsilon}$, and at $t=0$ we have

$$
2\left(H_{2}\right)_{t}(s, 0)=\tau_{g, 1} \tau_{n}+\tau_{g, 2}^{\prime}-2 H_{1} a_{12}^{3}+\bar{B}+\epsilon a \tau_{g, 2} .
$$

Therefore, assuming $a \not \equiv 0$, it results that

$$
\left.\frac{d}{d \epsilon}\left(\ln \pi^{\prime}(0)\right)\right|_{\epsilon=0}=\frac{1}{2} \int_{0}^{L} \frac{(a(s))^{2} \tau_{g, 2}(s)}{2 \tau_{g, 2}(s)} d s=\frac{1}{4} \int_{0}^{L}(a(s))^{2} d s \neq 0 .
$$

\section{Mean directional structural stability}

Let $I\left(M, \mathbb{R}^{4}\right)$ be the space of the immersions of $M$ into $\mathbb{R}^{4}$, where $M$ is a compact, smooth and oriented surface, endowed with the Whitney topology.

Lemma 5.1. Let $M$ be a surface which is the graph of the map $\alpha(u, v)=$ $(u, v, S(u, v), R(u, v))$, as in the Section 3. Suppose that $(0,0)$ is an $H$-singularity. We call $\beta_{a b}$ the immersion

$$
\beta(\alpha ; u, v ; a, b)=\left(u, v, S(u, v)+\frac{a}{2} u^{2}+\frac{b}{2} v^{2}, R(u, v)+a u v+\frac{b}{2} u^{2}\right),
$$

with $(a, b) \in V$, where $V$ is a neighborhood of $(0,0)$ and $(u, v) \in U$. Then there exists $D \subset U$ a compact disc on which

$$
a_{0}(u, v)=\frac{\partial(C, B)}{\partial(a, b)}(u, v ; 0,0) \neq 0,
$$

where $C$ and $B$ are obtained from the differential equation of mean directionally curved lines of $\beta_{a b}$.

Proof: The differential equation of mean directionally curved lines of $\beta_{a b}$ is given by

$$
C(u, v ; a, b)(d v)^{2}+2 B(u, v ; a, b) d u d v+A(u, v ; a, b)(d u)^{2}=0,
$$


where

$$
C=C(u, v ; a, b)=\left[2\left(f_{1} g_{2}-f_{2} g_{1}\right) F+\left(e_{2} g_{1}-e_{1} g_{2}\right) G\right](u, v ; a, b)
$$

and

$$
B=B(u, v ; a, b)=\left[\left(f_{1} g_{2}-f_{2} g_{1}\right) E+\left(e_{2} f_{1}-e_{1} f_{2}\right) G\right](u, v ; a, b) .
$$

The form of $A(u, v ; a, b)$ is not important here.

Without loss of generality we have:

1. If the $H$-singularity is a minimal point then through an appropriate rotation in the normal plane it is possible to consider the frame $\left\{N_{1}, N_{2}\right\}$ and the principal axes of the ellipse of curvature as being parallels. This implies that $e_{2}=g_{2}=f_{1}=0$ and $f_{2} g_{1} \neq 0$.

2. If the $H$-singularity is an inflection point then through an appropriate rotation in the normal plane it is possible to consider the ellipse of curvature on $N_{1}$-direction. This implies that $e_{2}=f_{2}=g_{2}=0$, $g_{1} \neq 0$ and $e_{1}+g_{1} \neq 0$.

From extensive calculation we have

$$
a_{0}(0,0)=\frac{\partial(C, B)}{\partial(a, b)}(0,0 ; 0,0)=\left(e_{2}+g_{1}\right)\left(e_{1}+g_{1}+f_{2}\right) \neq 0 .
$$

Therefore there exists $D \subset U$ a compact disc on which

$$
a_{0}(u, v)=\frac{\partial(C, B)}{\partial(a, b)}(u, v ; 0,0) \neq 0 .
$$

This ends the proof.

Lemma 5.2. Let $M$ be a surface which is the graph of the map $\alpha(u, v)=$ $(u, v, S(u, v), R(u, v))$. Suppose that $(0,0)$ is an $H$-singularity and let $\beta_{a b}$ be the immersion

$$
\beta(\alpha ; u, v ; a, b)=\left(u, v, S(u, v)+\frac{a}{2} u^{2}+\frac{b}{2} v^{2}, R(u, v)+a u v+\frac{b}{2} u^{2}\right),
$$

with $(a, b) \in V$, where $V$ is a neighborhood of $(0,0)$ and $(u, v) \in U$. Let $D \subset U$ be a compact disc on which

$$
a_{0}(u, v) \neq 0,
$$

as in the Lemma 5.1.

Call $V_{a b}=V_{a b}(D) \subset V$ the set of the pairs $(a, b)$ for which all $H$-singularities of the immersion $\beta_{a b}$, with $(u, v) \in D$, satisfy the transversality condition. There is a small $\rho>0$ such that the intersection of $V_{a b}$ with the disc of radius $\rho$ has full Lebesgue measure. 
Proof: The set

$$
S_{a b}=S\left(\beta_{a b}\right)=\left\{(u, v ; a, b) \in \mathbb{R}^{2} \times \mathbb{R}^{2} / C(u, v ; a, b)=0=B(u, v ; a, b)\right\}
$$

is the locus of points where the immersion $\beta_{a b}$ has $H$-singularities. Here $C$ and $B$ are the coefficients of the differential equation of mean directionally curved lines of $\beta_{a b}$.

At transversal $H$-singularities $S_{a b}$ is a smooth surface, since at these points

$$
\frac{\partial(C, B)}{\partial(u, v)} \neq 0 .
$$

Now we prove that if $(a, b)$ is close to $(0,0)$, the surface $S_{a b}$ is regular even at non transversal $H$-singularities.

From the Lemma 5.1, there exists $D \subset U$ a compact disc on which

$$
a_{0}(u, v)=\frac{\partial(C, B)}{\partial(a, b)}(u, v ; 0,0) \neq 0 .
$$

This shows that there exists a neighborhood $V_{a b}=\{|(a, b)|<\rho\}$ such that if $(u, v) \in D$ and $(a, b) \in V_{a b}$ then

$$
\frac{\partial(C, B)}{\partial(a, b)}(u, v ; a, b) \neq 0 .
$$

This concludes the proof of the smoothness of $S_{a b}$. Now the lemma is a consequence of Sard's Theorem, by the identification of $V_{a b}$ with the regular values of the orthogonal projection of $S_{a b}$ to the $a b$-plane.

Theorem 5.3. The set $\alpha \in I\left(M, \mathbb{R}^{4}\right)$ of immersions such that all $H$-singularities are of $S$-type is open and dense in $I\left(M, \mathbb{R}^{4}\right)$.

Proof: The conditions imposed to the immersion $\alpha$ near an $H$-singularity of $S$-type depend on the derivatives of order three and are open, so it implies that this set is open.

If the transversality condition holds for an $H$-singularity, a small perturbation on the parameters of the coordinate chart defines an $H$-singularity of $S$-type. Thus it is enough to consider $H$-singularities for which the transversality condition holds.

The set of $H$-singularities of an immersion $\alpha$ is compact, therefore it can be covered by a finite number of Monge charts. Using the local Lemma 5.2, which can be globalized by a standard argument, the theorem is proved. 
An immersion $\alpha$ is said to be mean directional stable if it has a neighborhood $V(\alpha)$, such that for any $\beta \in V(\alpha)$ there exists a homeomorphism $h: M \rightarrow M$ mapping $S(\alpha)$ onto $S(\beta)$, mapping $F_{H}(\alpha)$ onto $F_{H}(\beta)$ and mapping $f_{H}(\alpha)$ onto $f_{H}(\beta)$.

Consider the subset $\Sigma \subset I\left(M, \mathbb{R}^{4}\right)$ of immersions $\alpha$ defined by the following conditions:

1. All $H$-singularities are $S$-type (Section 3 ).

2. All mean directionally curved cycles are hyperbolic (Section 4).

3 . The limit set of every mean directionally curved line is contained in the set of $\mathrm{H}$-singularities and mean directionally curved cycles.

4. There are no connections or self connections of $H$-singularity separatrices (Section 3).

Theorem 5.4. The set $\Sigma$ is open in $I\left(M, \mathbb{R}^{4}\right)$ and every $\alpha \in \Sigma$ is mean directional stable.

Proof: We take on the projective bundle $P M$, as in Section 3, the surface $W_{\alpha}$ defined by the differential equation of mean directionally curved lines $(2.2)$. In coordinates $(u, v ; p)$ this surface is defined by $W_{\alpha}=T_{\alpha}^{-1}(0)$, where $T_{\alpha}(u, v ; p)=C(u, v) p^{2}+2 B(u, v) p+A(u, v)$. This surface is regular under the $H$-singularity hypothesis. The restriction of the natural projection $\pi: P M \rightarrow M$ to $W_{\alpha}$ is a double covering outside the preimage of the set $S(\alpha)$.

On $P M$ we define the involution $I(u, v ;[d u: d v])=(u, v ;[d v:-d u])$ which amounts to a rotation of lines by an angle $\pi / 2$. The surface $W_{\alpha}$ is invariant under $I$. On $W_{\alpha}-\pi^{-1}(S(\alpha))$ we define the vector field $X_{\alpha}$, which in the coordinates $(u, v ; p)$ has the form $X_{\alpha}=\left(\left(T_{\alpha}\right)_{p}, p\left(T_{\alpha}\right)_{p},-\left(\left(T_{\alpha}\right)_{u}+\right.\right.$ $\left.\left.p\left(T_{\alpha}\right)_{v}\right)\right)$. This vector field has an unique regular extension to $\pi^{-1}(S(\alpha))$.

We consider the induced line field $I_{*} X_{\alpha}$. Thus it is obtained a transversal pair $\left\{X_{\alpha}, I_{*} X_{\alpha}\right\}$ on $W_{\alpha}-\pi^{-1}(S(\alpha))$. Therefore we have defined a net outside $\pi^{-1}(S(\alpha))$, with the following properties: this net is invariant under $I$ and by $\pi$ projects to the net $\left(F_{H}(\alpha), f_{H}(\alpha)\right)$.

With the above constructions this situation is connected with the case of the principal line fields and their canonical regions [11]. Thus the construction and continuation to a small neighborhood $V(\alpha)$ of $\alpha$ of canonical regions follow also from the openness and unique continuation, for $\beta$ near $\alpha$, of singularities and of cycles due to the hyperbolicity of these elements in the fields of the pair $\left\{X_{\alpha}, I_{*} X_{\alpha}\right\}$. This leads of the openness of $\Sigma$ and gives uniquely a correspondence between $H$-singularities, separatrices, cycles and their intersections for $\left\{X_{\alpha}, I_{*} X_{\alpha}\right\}$ and 
$\left\{X_{\beta}, I_{*} X_{\beta}\right\}$. The extension of this correspondence to a topological equivalence $H: W_{\alpha} \rightarrow W_{\beta}$ which, by projection, gives the topological equivalence $h: M \rightarrow M$ between $\mathcal{H}(\alpha)$ and $\mathcal{H}(\beta)$ is carried out as in the case of nets of asymptotic lines on surfaces immersed in $\mathbb{R}^{3}$, according to $[\mathbf{4}]$.

\section{A special case}

Let $\alpha: M \rightarrow S^{3}$ be an immersion of a smooth and oriented surface into $S^{3}$. Consider the natural inclusion $i: S^{3} \rightarrow \mathbb{R}^{4}$ and the composition $\alpha=i \circ \alpha$ still denoted by $\alpha$. Assume that $(u, v)$ is a positive chart of $M$ and that $\left\{\alpha_{u}, \alpha_{v}, N_{1}, N_{2}\right\}$ is a positive frame of $\mathbb{R}^{4},\left\{N_{1}, N_{2}\right\}$ being a frame of vector fields orthonormal to $\alpha$, where $N_{1}(p) \in T_{p} S^{3}$ and $N_{2}(p)$ is the inward normal to $S^{3}$, for all $p \in M$. Thus $N_{2} \equiv-\alpha$.

In such a chart $(u, v)$

$$
e_{2}=E, \quad f_{2}=F \quad \text { and } \quad g_{2}=G,
$$

where $E, F$ and $G$ are the coefficients of the first fundamental form of $\alpha$. It follows that $I I_{2}=I$. Now

$$
\eta=\frac{I I}{I}=\frac{I I_{1}}{I} N_{1}+\frac{I I_{2}}{I} N_{2}=\frac{I I_{1}}{I} N_{1}+N_{2} .
$$

This implies that the ellipse of curvature is degenerate as a line segment on $N_{2}=1$, for all $p \in M$. In classic literature, this type of points are called semiumbilics and this result has been already obtained in [14]. We have

$$
H_{2}=\frac{E g_{2}-2 F f_{2}+G e_{2}}{2\left(E G-F^{2}\right)}=1,
$$

for all $p \in M$. It follows that $H(p) \neq 0$, for all $p \in M$. So, if $p$ is an $H$-singularity of $M$ then $p$ is an inflection point of $M$. In this point the ellipse of curvature becomes a point.

As an example consider the following construction. Let $\phi: \mathbb{R}^{3} \rightarrow S^{3} \subset$ $\mathbb{R}^{4}$ be the stereographic projection given by

$$
\phi(x, y, z)=\frac{1}{1+w}(x, y, z, w),
$$

where $w=\frac{1}{2}\left(x^{2}+y^{2}+z^{2}-1\right)$. We recall that $\phi$ is conformal.

Let $\alpha: M \rightarrow \mathbb{R}^{3}$ be an immersion of a smooth and oriented surface $M$ into $\mathbb{R}^{3}$. Assume that $(u, v)$ is a positive chart of $M$ and that $\left\{\alpha_{u}, \alpha_{v}, N\right\}$ is a positive frame of $\mathbb{R}^{3}$, where

$$
N=\frac{\alpha_{u} \wedge \alpha_{v}}{\left\|\alpha_{u} \wedge \alpha_{v}\right\|}
$$


is the normal vector field to $\alpha$. Let $\bar{\alpha}=\phi \circ \alpha$ be the stereographic projection of $M$ in $S^{3}$ and let $\bar{\alpha}=i \circ \bar{\alpha}$ be the immersion of $M$ into $\mathbb{R}^{4}$, where $\left\{\bar{\alpha}_{u}, \bar{\alpha}_{v}, N_{1}, N_{2}\right\}$ is a positive frame of $\mathbb{R}^{4}$, being

$$
N_{1}=\frac{d \phi(N)}{\|d \phi(N)\|}
$$

and $N_{2}$ the inward unitary normal to $S^{3}$.

From extensive calculation

$$
\begin{aligned}
& \bar{E}=(1+w)^{-2} E, \\
& \bar{F}=(1+w)^{-2} F, \\
& \bar{G}=(1+w)^{-2} G, \\
& \overline{e_{1}}=(1+w)^{-2}[(1+w) e+E t], \\
& \overline{f_{1}}=(1+w)^{-2}[(1+w) f+F t], \\
& \overline{g_{1}}=(1+w)^{-2}[(1+w) g+G t], \\
& \overline{e_{2}}=\bar{E}, \\
& \overline{f_{2}}=\bar{F}, \\
& \overline{g_{2}}=\bar{G},
\end{aligned}
$$

and

where the expressions without (with respectively) bar are associated to $\alpha$ ( $\bar{\alpha}$ respectively), and $t=\langle\alpha, N\rangle$ is the support function of $\alpha$.

Lines of axial curvature on surfaces immersed in $\mathbb{R}^{4}$ are lines along which the second fundamental form points in the direction of principal axes of the ellipse of curvature. The differential equation of lines of axial curvature is given by $[\mathbf{6}]$,

$$
\operatorname{Jac}\left(\|\eta-H\|^{2}, I\right)=0,
$$

which is a quartic differential equation.

Asymptotic lines on surfaces immersed in $\mathbb{R}^{4}$ are lines along which the second fundamental form points in the direction of the tangent lines to the ellipse of curvature. The differential equation of asymptotic lines is given by

$$
\operatorname{Jac}\left(I I_{1}, I I_{2}\right)=0 .
$$


Remark 6.1. Through the above construction lines of principal curvature of $\alpha$ are carried over into asymptotic lines of $\bar{\alpha}$, lines of mean curvature of $\alpha$ are carried over into mean directionally curved lines of $\bar{\alpha}$ and umbilic points of $\alpha$ are carried over into inflection points of $\bar{\alpha}$. These results are also presented in $[\mathbf{1 2}]$ and $[\mathbf{1 3}]$.

Examples of immersions $\beta \in \Sigma$ can be obtained from the Remark 6.1. Consider the set $\Psi$ of immersions of surfaces into $\mathbb{R}^{3}$ where every $\alpha \in \Psi$ is mean curvature structurally stable, according to [7]. Thus if $\alpha \in \Psi$ then $\bar{\alpha} \in \Sigma$, where $\bar{\alpha}=i \circ \phi \circ \alpha$ is as above.

Let $\alpha: M \rightarrow \mathbb{R}^{4}$ be an immersion of a smooth and oriented surface into $\mathbb{R}^{4}$. The quartic differential equation (6.1) can be written as the product of two quadratic differential equations if the image of the surface $M$ by $\alpha$ is contained into $\mathbb{R}^{3}$, according to [6]. We have the following theorem.

Theorem 6.2. Let $\alpha: M \rightarrow S^{3}$ be an immersion of a smooth and oriented surface into $S^{3}$. Consider the natural inclusion $i: S^{3} \rightarrow \mathbb{R}^{4}$ and the composition $\alpha=i \circ \alpha$ still denoted by $\alpha$. Then the quartic differential equation (6.1) can be written as

$$
\operatorname{Jac}\left\{\operatorname{Jac}\left(I I_{1}, I\right), I\right\} \operatorname{Jac}\left(I I_{1}, I\right)=0,
$$

where the first expression in (6.3) is the quadratic differential equation of mean directionally curved lines (2.6) and the second one is the quadratic differential equation of asymptotic lines (6.2).

Proof: From the coefficients of the first and the second fundamental forms listed above write the product $\operatorname{Jac}\left\{\operatorname{Jac}\left(I I_{1}, I\right), I\right\} \operatorname{Jac}\left(I I_{1}, I\right)$ and compare the result with $\operatorname{Jac}\left(\|\eta-H\|^{2}, I\right)$. The theorem is proved.

\section{References}

[1] A. C. Asperti, Immersions of surfaces into 4-dimensional spaces with nonzero normal curvature, Ann. Mat. Pura Appl. (4) 125 (1980), 313-328.

[2] J. W. Bruce and D. L. Fidal, On binary differential equations and umbilics, Proc. Roy. Soc. Edinburgh Sect. A 111(1-2) (1989), $147-168$.

[3] A. R. Fonsyth, "Geometry of four dimensions", vols. I and II, Cambridge Univ. Press, Cambridge, 1930.

[4] R. Garcia, C. Gutierrez and J. Sotomayor, Structural stability of asymptotic lines on surfaces immersed in $\mathbb{R}^{3}$, Bull. Sci. Math. 123(8) (1999), 599-622. 
[5] R. Garcia, D. K. H. Mochida, M. C. Romero-Fuster and M. A. S. RuAS, Inflection points and topology of surfaces in 4-space, Trans. Amer. Math. Soc. 352(7) (2000), 3029-3043.

[6] R. Garcia and J. Sotomayor, Lines of axial curvature on surfaces immersed in $\mathbb{R}^{4}$, Differential Geom. Appl. 12(3) (2000), 253-269.

[7] R. Garcia And J. Sotomayor, Structurally stable configurations of lines of mean curvature and umbilic points on surfaces immersed in $\mathbb{R}^{3}$, Publ. Mat. 45(2) (2001), 431-466.

[8] I. Guadalupe, C. Gutierrez, J. Sotomayor and R. Tribuzy, Principal lines on surfaces minimally immersed in constantly curved 4-spaces, in: "Dynamical systems and bifurcation theory" (Rio de Janeiro, 1985), Pitman Res. Notes Math. Ser. 160, Longman Sci. Tech., Harlow, 1987, pp. 91-120.

[9] C. Gutierrez, I. Guadalupe, R. Tribuzy and V. Guíñez, Lines of curvature on surfaces immersed in $\mathbb{R}^{4}$, Bol. Soc. Brasil. Mat. (N.S.) 28(2) (1997), 233-251.

[10] C. Gutierrez and J. Sotomayor, An approximation theorem for immersions with stable configurations of lines of principal curvature, in: "Geometric dynamics" (Rio de Janeiro, 1981), Lecture Notes in Math. 1007, Springer, Berlin, 1983, pp. 332-368.

[11] C. Gutierrez and J. Sotomayor, Lines of curvature and umbilical points on surfaces, 18th Brazilian Math. Colloquium, IMPA, Rio de Janeiro (1991); reprinted as: Structurally stable configurations of lines of curvature and umbilic points on surfaces, Monografías del IMCA, Lima (1998).

[12] J. A. LitTle, On singularities of submanifolds of higher dimensional Euclidean spaces, Ann. Mat. Pura Appl. (4) 83 (1969), 261-335.

[13] D. K. H. Mochida, M. C. Romero-Fuster and M. A. S. Ruas, Osculating hyperplanes and asymptotic directions of codimension two submanifolds of Euclidean spaces, Geom. Dedicata 77(3) (1999), 305-315.

[14] M. C. Romero-Fuster and F. SÁnchez-Bringas, Umbilicity of surfaces with orthogonal asymptotic lines in $\mathbb{R}^{4}$, Differential Geom. Appl. 16(3) (2002), 213-224.

[15] F. SÁnchez-Bringas and A. I. Ramírez-Galarza, Lines of curvature near umbilical points on surfaces immersed in $\mathbb{R}^{4}$, Ann. Global Anal. Geom. 13(2) (1995), 129-140. 
[16] J. Sotomayor and C. Gutierrez, Structurally stable configurations of lines of principal curvature, in: "Bifurcation, ergodic theory and applications" (Dijon, 1981), Astérisque 98-99, Soc. Math. France, Paris, 1982, pp. 195-215.

[17] M. SpIVAK, "A comprehensive introduction to differential geometry", vol. IV, second edition, Publish or Perish, Inc., Wilmington, Del., 1979.

[18] W. C. Wong, A new curvature theory for surfaces in Euclidean 4-spaces, Comment. Math. Helv. 26 (1952), 152-170.

Instituto de Ciências

Universidade Federal de Itajubá

CEP 37.500-000, Itajubá, MG

Brazil

E-mail address: lfmelo@unifei.edu.br

Primera versió rebuda el 20 de setembre de 2002, darrera versió rebuda el 25 de novembre de 2002. 